\title{
The spectrum of eye disease in hospitalized adults living with HIV, 1995-2010.
}

\author{
Christopher Miller \\ Division of Infectious Diseases, Thomas Jefferson University Hospital \\ William Short, MD, MPH \\ Division of Infectious Diseases, Thomas Jefferson University Hospital; Jefferson Medical College, \\ Thomas Jefferson University \\ Lorena Perez-Povis \\ Division of Infectious Diseases, Thomas Jefferson University Hospital; Shannon Clinic and Shannon \\ Medical Center \\ Josephine Lontok

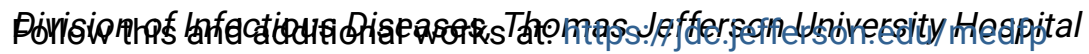

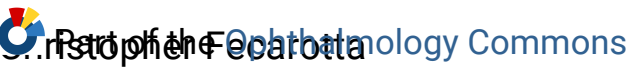

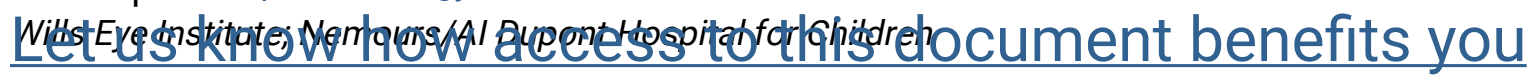

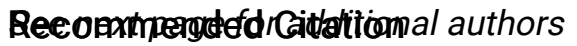

Miller, Christopher; Short, MD, MPH, William; Perez-Povis, Lorena; Lontok, Josephine; Fecarotta, Christopher; Liu, Mengdan; Sendecki, Jocelyn; and Belden, Katherine, "The spectrum of eye disease in hospitalized adults living with HIV, 1995-2010." (2014). Department of Medicine Faculty Papers. Paper 123.

https://jdc.jefferson.edu/medfp/123

This Article is brought to you for free and open access by the Jefferson Digital Commons. The Jefferson Digital Commons is a service of Thomas Jefferson University's Center for Teaching and Learning (CTL). The Commons is a showcase for Jefferson books and journals, peer-reviewed scholarly publications, unique historical collections from the University archives, and teaching tools. The Jefferson Digital Commons allows researchers and interested readers anywhere in the world to learn about and keep up to date with Jefferson scholarship. This article has been accepted for inclusion in Department of Medicine Faculty Papers by an authorized administrator of the Jefferson Digital Commons. For more information, please contact: JeffersonDigitalCommons@jefferson.edu. 


\section{Authors}

Christopher Miller; William Short, MD, MPH; Lorena Perez-Povis; Josephine Lontok; Christopher Fecarotta; Mengdan Liu; Jocelyn Sendecki; and Katherine Belden 


\title{
The Spectrum of Eye Disease in Hospitalized Adults Living with HIV, 1995-2010
}

\author{
Christopher Miller, MD, William R. Short, MD, MPH, ${ }^{1,6}$ Lorena Perez-Povis, MD,,2 Josephine Lontok, MD, \\ Christopher Fecarotta, MD, ${ }^{3}$ Mengdan Liu, ${ }^{4}$ Jocelyn Sendecki, ${ }^{5}$ and Katherine Belden, MD ${ }^{1,6}$
}

\begin{abstract}
Eye disease is a well-documented complication of HIV infection. Opportunistic infections generally comprised the majority of pre-antiretroviral therapy (ART) eye complications. With the introduction of ART, opportunistic infections diminished. However, early ART regimens were cumbersome regarding side effects and pill burden, making patient compliance difficult. Newer ART regimens are better tolerated and consist of fewer pills, theoretically making compliance easier and therapy more effective. The aim of this chart review study is to examine eye disease epidemiology in HIV patients as ART has evolved. We reviewed 222 admissions at Thomas Jefferson University Hospitals for 188 patients. These cases were divided into two groups. The first group was comprised of patients admitted from 1995 through 2003, while the second group consisted of patients admitted from 2003 to 2010. Eye disease epidemiology was compared between the two groups. Our study did note a significant decrease in eye diseases caused by opportunistic infections in the 2003-2010 patient group. Noninfectious eye disease is a significant complication in this group.
\end{abstract}

\section{Introduction}

$\mathbf{I}^{\mathrm{N}}$ N THE PRE-HIGHLY ACTIVE ANTIRETROVIRAL THERAPY ERA (HAART, or ART; please note that HAART serves as a historical term for this article. We will refer to such HIV treatment regimens as "antiretroviral therapy" or ART), opportunistic infections of the eye were frequent and often severe. ${ }^{1}$ However, the epidemiology of eye involvement in HIV has changed as a result of our ability to control HIV disease with newer ART regimens. ${ }^{2-7}$ The incidence and overall severity of opportunistic infectious diseases of the eye have decreased, along with other sequelae of immunosuppression.

The stark contrast in HIV eye diseases before and after the introduction of ART has been described in the literature. $^{8}$ However, epidemiologic differences in HIV eye disease that have occurred during the ART era have not been as well established. Recent studies still estimate that approximately $40 \%$ of patients with AIDS and no evidence of infectious retinitis still demonstrate significant visual impairment. ${ }^{9}$ Such ongoing disease may be indirectly due to a persistent systemic inflammatory state (the hypothesized etiology of HIV retinopathy) or due to the aforementioned increase in incidence of additional non-AIDS related comorbidities in today's HIV patients (diabetes or hypertension, for instance).

The aim of this study is to review retrospectively a population of HIV-positive hospitalized patients with eye disease over this time period of ART evolution. We then compare the epidemiology of eye disease in patients seen in the early era of ART with the more recent era of ART.

\section{Methods}

\section{Patient population}

HIV-infected adults with active ocular disease admitted as inpatients for any reason to the Wills Eye Institute and Thomas Jefferson University Hospitals between December 30, 1995 and October 31, 2010 were identified through a search of applied International Classification of Diseases, Ninth Revision, Clinical Modification (ICD-9) codes including HIV (042, V08) and any ocular diagnosis (360-379).

An initial 422 admissions were identified. Of these, 9 charts were unavailable, 129 admissions were noted to have no active ocular diagnosis, 59 were noted to be duplicate patient

\footnotetext{
Divisions of ${ }^{1}$ Infectious Diseases and ${ }^{5}$ Biostatistics, Thomas Jefferson University Hospital, Philadelphia, Pennsylvania.

${ }^{2}$ Shannon Clinic and Shannon Medical Center, San Angelo, Texas.

${ }^{3}$ Wills Eye Institute, Philadelphia, Pennsylvania; and Nemours/AI Dupont Hospital for Children, Wilmington, Delaware.

${ }^{4}$ Center for Research in Medical Education and Health Care, Jefferson Medical College, Philadelphia, Pennsylvania.

${ }^{6}$ Jefferson Medical College of Thomas Jefferson University.
} 
TABle 1. Demographics ${ }^{\mathrm{a}}$

\begin{tabular}{|c|c|c|c|c|}
\hline Demographics & All patients $(\mathrm{n}=188)$ & Early ART $(\mathrm{n}=60)$ & Late ART $(\mathrm{n}=128)$ & $\mathrm{p}$ Value \\
\hline Age (yrs) (median) & 50 & 51 & 50 & 0.2491 \\
\hline Gender ( $\%$ male $)$ & 68.6 & 73.3 & 66.4 & 0.34 \\
\hline Race $(\%)$ & & & & 0.032 \\
\hline White non-hispanic & 26 & 15 & 31.2 & \\
\hline Black non-hispanic & 68.6 & 81.7 & 62.4 & \\
\hline Other & 5.4 & 3.3 & 6.4 & \\
\hline Substance abuse (\%) & $32.8, n=183$ & $31.6, n=57$ & $33.3, n=126$ & 0.8149 \\
\hline Tobacco abuse (\%) & $29, n=183$ & $26.3, n=57$ & $30.2, n=126$ & 0.5956 \\
\hline \multicolumn{5}{|l|}{ HIV history } \\
\hline Median time (yrs) since HIV diagnosis & $6, n=85$ & $6, n=39$ & $7.5, n=46$ & 0.0673 \\
\hline Median CD4 + T-cells (cells $/ \mu \mathrm{L})$ & $83, n=143$ & $40, n=50$ & $108, n=93$ & $<0.0001$ \\
\hline$\%$ CD $4+$ T-cells $<200$ cells $/ \mu \mathrm{L}$ & $72, n=143$ & $88, n=50$ & $63.4, n=93$ & 0.0052 \\
\hline$\%$ Patients with HIV viral load $>400$ copies $/ \mathrm{mL}$ & $72.1, n=86$ & $88.5, n=26$ & $65, n=60$ & 0.0259 \\
\hline Current antiretroviral therapy $(\%)$ & 58.5 & 51.7 & 61.7 & 0.1922 \\
\hline History of opportunistic infection & $47.4, n=175$ & $72.2, n=54$ & $36.4, n=121$ & $<0.0001$ \\
\hline Selected co-morbidities (\%) & $N=183$ & $N=57$ & $N=126$ & \\
\hline Diabetes mellitus & 13.7 & 7 & 16.7 & 0.0784 \\
\hline Malignancy & 11.5 & 1.8 & 15.9 & 0.0055 \\
\hline Cardiovascular disease & 20.8 & 15.8 & 23 & 0.2644 \\
\hline Chronic kidney disease & 8.2 & 8.8 & 7.9 & 1 \\
\hline Chronic viral hepatitis & 20.8 & 17.5 & 22.2 & 0.47 \\
\hline
\end{tabular}

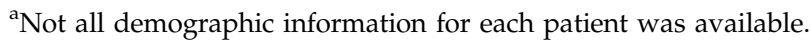

admissions without a new ocular diagnosis, and 3 admissions did not have confirmed HIV by history or laboratory testing. These cases were excluded, leaving 222 admissions amongst 188 patients.

A retrospective chart review was conducted. Data for the study were collected from included charts. Demographic patient information collected included age, gender, race, substance abuse history, CD $4+\mathrm{T}$ cells $/ \mu \mathrm{L}$, HIV viral load, current antiretroviral therapy, and select co-morbidities (Table 1).

\section{Outcome}

Active ocular diagnoses were recorded for each admission. Visual acuity was documented when available (160 of 188 patients had a full ophthalmologic exam with full visual acuity). All patients in the study with documented visual acuity received a full ophthalmologic exam. For the 22 patients without documented visual acuity, documentation of further ophthalmologic examination was not recorded.

Patients and their active ocular diagnoses were divided into comparison groups based on date of presentation. An "Early ART" arm of the study consisted of presentations from December 30, 1995 through June 30, 2003. A "Late ART" arm of the study consisted of presentations from July 1, 2003 through October 31, 2010.

For this study, 2003 was chosen as a separation time point between study groups. Prior to 2003, protease inhibitor (PI) regimens still proved cumbersome and triple-nucleotide reverse transcriptase inhibitor (NRTI) regimens still remained in use. In 2003 and beyond, newer, better-tolerated medications such as atazanavir, fosamprenavir, and fixed-dose combination pills such as tenofovir/emtricitabine and tenofovir/ emtricitabine/efavirenz became available. Therefore, 2003 seemed a reasonable time to demarcate an "early" time frame capturing patients on first-generation PIs and NRTIs versus a "late" time frame capturing patients on modern PIs, fixeddose combination pills, and NNRTIs.

\section{Statistics}

$\mathrm{p}$ Values were obtained with the unpaired $t$-test, chi-square test, or Fisher's exact test and adjusted by false discovery rate methods, when indicated.

\section{Results}

From the 222 admissions, 252 active ocular diagnoses were identified (please refer to Table 4 for a complete breakdown by anatomic location). Sixty patients were included in the early ART arm of the study, and 128 patients were included in the late ART arm (Table 1).

Immunologic and virologic comparison of the two patient groups illustrated a significantly higher median CD4 + count in the late ART patients compared with the early ART patients (108 vs. 40 cells $/ \mu \mathrm{L}$ ). The late ART group also had significantly fewer patients with uncontrolled viral load (defined as $>400$ copies $/ \mathrm{mL}$ ) compared with the early ART group (65\% vs. $88.5 \%$ of patients). In addition, late ART patients were less likely to have had an opportunistic infection in the past $(36.4 \%$ of late ART patients had a history vs. $72.2 \%$ of early ART patients).

TABle 2. Infectious vs. Noninfectious Ocular Diagnoses

\begin{tabular}{lcccc}
\hline & $\begin{array}{c}\text { Total } \\
\text { diagnoses (\%) } \\
\mathrm{N}=252\end{array}$ & $\begin{array}{c}\text { Early } \\
\text { ART (\%) } \\
\mathrm{N}=77\end{array}$ & $\begin{array}{c}\text { Late } \\
\text { ART (\%) } \\
\mathrm{N}=175\end{array}$ & $\mathrm{p}$ Value \\
\hline $\begin{array}{c}\text { Infectious } \\
\text { diagnosis } \\
\begin{array}{c}\text { Noninfectious } \\
\text { diagnosis }\end{array}\end{array}$ & 43 & 54.6 & 37.7 & 0.0129 \\
\hline
\end{tabular}


Table 3. Most Frequent Ocular Diagnoses By Early and Late ART Groups

\begin{tabular}{|c|c|c|c|c|}
\hline Diagnosis & $\begin{array}{l}\text { Total diagnoses (\%) } \\
\qquad \mathrm{N}=252\end{array}$ & $\begin{array}{l}\text { Early ART (\%) } \\
\qquad \mathrm{N}=77\end{array}$ & $\begin{array}{l}\text { Late ART }(\%) \\
\quad \mathrm{N}=175\end{array}$ & p Value \\
\hline Noninfectious retinopathy & 13.9 & 18.2 & 12 & 0.4097 \\
\hline CMV retinitis (Fig. 1) & 13.9 & 28.6 & 7.4 & $<0.0001$ \\
\hline Retinal detachment (Fig. 4) & 7.6 & 6.5 & 8 & 1 \\
\hline Ulcerative keratitis & 7.1 & 3.9 & 8.6 & 0.4097 \\
\hline Necrotizing herpetic retinitis/viral retinitis (Fig. 2) & 5.6 & 5.2 & 6.3 & 1 \\
\hline Preseptal cellulitis & 3.6 & 3.9 & 3.4 & 1 \\
\hline Trauma & 3.2 & 0 & 4.6 & 0.4097 \\
\hline Cataract & 2.8 & 0 & 4 & 0.4097 \\
\hline Ocular syphilis & 2.8 & 2.6 & 2.9 & 1 \\
\hline Cranial nerve palsy & 2.8 & 0 & 4 & 0.4097 \\
\hline Conjunctivitis & 2.4 & 2.6 & 2.3 & 1 \\
\hline Conjunctival hemorrhage & 2.4 & 0 & 3.4 & 0.4097 \\
\hline Glaucoma & 2.4 & 1.3 & 2.9 & 1 \\
\hline Noninfectious uveitis & 2.4 & 3.9 & 1.7 & 0.7003 \\
\hline Endogenous endophthalmitis & 1.9 & 1.3 & 2.3 & 1 \\
\hline Optic neuritis & 1.9 & 3.9 & 1.1 & 0.4097 \\
\hline
\end{tabular}

The late ART group of patients, however, tended to have more co-morbidities compared to the early ART patients. Most significantly, patients in the late ART group had a 15.9\% prevalence of malignancy versus only a $1.8 \%$ incidence in the early ART group. Late ART patients also had a significantly higher prevalence of diabetes mellitus compared to their early ART counterparts ( $16.7 \%$ vs. $7 \%$ prevalence). While not statistically significant, late ART patients also tended to have a higher prevalence of cardiovascular disease and chronic viral hepatitis (Table 1).

Among the early ART patients, 77 ocular diseases were diagnosed, while 175 ocular diseases were diagnosed in the late ART group. Ocular disease in both groups combined was predominately noninfectious (57\%). Ocular diagnoses were compared in early and late ART groupings. Early ART diagnoses had significantly more infectious eye disease occurrence compared to the late ART group (54.6\% of cases vs. $37.7 \%$ ) (Table 2).

The most frequent ocular diagnoses (from Table 4) found in the early and late ART groups were compared with $p$ values calculated for the differences in prevalence (Table 3) (note that $p$ values were not calculated for differences in all diagnoses from Table 4, just for the most frequent that appear in Table 3). Most notably, $28.6 \%$ of ocular disease in the early ART group was CMV retinitis (Fig. 1) compared to only $7.4 \%$ in the late ART group. This diagnosis represented the highest burden of ocular disease in early ART patients by a large margin. In contrast, the incidence of ocular disease diagnoses in the late ART group was more evenly distributed amongst a wide range of categories.

The late ART group did have a significantly higher rate of severe vision impairment, however, when visual acuity was documented. Forty-eight patients in the early ART group and 112 patients in the late ART group had visual acuity documented. While the percentage of patients with "mild" visual impairment ( $<20 / 40$ but $\geq 20 / 200)$ was roughly equal $(22.9 \%$ of early ART patients vs. $19.6 \%$ of late ART patients, $p$ value of 0.64$)$, the percentage of patients with "severe" visual impairment $(<20 / 200)$ was significantly more for the late ART group (37.5\% of early ART patients versus $58 \%$ of late ART patients, $p$ value of 0.02 ).

\section{Discussion}

Ocular disease is associated with human immunodeficiency virus (HIV) infection. ${ }^{10-12}$ Ocular manifestations of HIV infection have been well described in the pre-ART era of HIV infection when patients frequently developed severe immunodeficiency followed by the development of opportunistic infections. Direct therapy (ganciclovir for cytomegalovirus, or CMV retinitis, for instance) for such opportunistic infections provided limited benefit in patients suffering from a progressively failing immune system. ${ }^{13}$

With the approval of zidovudine as the first antiretroviral therapy for HIV in 1987, the face of HIV disease began to change. The most dramatic change came in the mid-1990s with the introduction of ART, which made preservation of T-cell immunity and suppression of HIV possible. Early ART regimens, however, were inconvenient and cumbersome for patients due to high pill burdens, food restrictions, and intolerable side effects. ${ }^{2-7}$ Drug compliance, and therefore control of HIV disease, remained a challenge. With time, however, drugs with better tolerability, potency, and efficacy have been developed. Such developments have even made it possible to include a complete ART regimen co-formulated into one pill taken once daily. As a result, compliance and control of HIV are now much more realistic goals.

Eye disease caused by infectious agents such as Toxoplasma gondii (Fig. 5), varicella-zoster virus (VZV) (Fig. 3), and, most notably, CMV (Fig. 1), were disproportionately represented in HIV patients before HAART became widely available. One autopsy study from the pre-ART era found a $32 \%$ incidence of postmortem HIV-infected patients with evidence of CMV retinitis, ${ }^{14}$ and that the incidence of clinically apparent $\mathrm{CMV}$ retinitis in individuals with severe T-cell impairment (CD4+ $<100$ cells $/ \mu \mathrm{L}$ ) may be as high as $25 \% .{ }^{15}$ Frequently, HIVinfected patients with CMV retinitis progressed to retinal detachment and severe visual impairment months after the onset of visual disturbance.

As control of HIV has improved, the incidence of opportunistic infections decreased. ${ }^{2-7,11}$ One epidemiologic study from 1996 to 2004 found a 92\% decrease in AIDS-related mortality in HIV patients while ART utilization increased from $43 \%$ to $82 \%$ 
Table 4. Active Ocular Diagnoses by Anatomic Location

\begin{tabular}{|c|c|c|c|}
\hline Eye anatomic location & All diagnoses $(\mathrm{n}=252)$ & Early $A R T(\mathrm{n}=77)$ & Late ART $(\mathrm{n}=175)$ \\
\hline Adnexa/orbit & $44(17.4 \%)$ & 10 & 34 \\
\hline$\overline{\text { Eyelids }}$ & $15(5.9 \%)$ & 5 & 10 \\
\hline Preseptal cellulitis & $9(3.6 \%)$ & 3 & 6 \\
\hline Blepharitis (infectious) & $3(1.2 \%)$ & 2 & 1 \\
\hline Ectropion & $2(0.8 \%)$ & 0 & 2 \\
\hline Entropion & $1(0.4 \%)$ & 0 & 1 \\
\hline Orbit and surrounding tissues & $11(4.4 \%)$ & 1 & 10 \\
\hline Ocular invasive tumor & $4(1.6 \%)$ & 0 & 4 \\
\hline Orbital cellulitis & $3(1.2 \%)$ & 1 & 2 \\
\hline Thyroid ophthalmopathy/exophthalmos & $2(0.8 \%)$ & 0 & 2 \\
\hline Infected eye implant & $1(0.4 \%)$ & 0 & 1 \\
\hline Non-infectious frozen globe & $1(0.4 \%)$ & 0 & 1 \\
\hline Eyelid/orbit & $8(3.2 \%)$ & 0 & 8 \\
\hline Trauma & $8(3.2 \%)$ & 0 & 8 \\
\hline Lacrimal ducts & $6(2.4 \%)$ & 4 & 2 \\
\hline Dry eye syndrome & $4(1.6 \%)$ & 4 & 0 \\
\hline Dacryostenosis & $1(0.4 \%)$ & 0 & 1 \\
\hline Dacryostenosis (infectious) & $1(0.4 \%)$ & 0 & 1 \\
\hline Combined & $4(1.6 \%)$ & 0 & 4 \\
\hline Herpes zoster ophthalmicus & $4(1.6 \%)$ & 0 & 4 \\
\hline Anterior segment disease & $59(23.4 \%)$ & 11 & 48 \\
\hline Cornea & $24(9.5 \%)$ & 5 & 19 \\
\hline Ulcerative keratitis & $18(7.1 \%)$ & 3 & 15 \\
\hline Noninfectious & $7(2.8 \%)$ & 2 & 5 \\
\hline Infectious & $11(4.4 \%)$ & 1 & 10 \\
\hline Non-ulcerative keratitis & $3(1.2 \%)$ & 2 & 1 \\
\hline Corneal scar & $2(0.8 \%)$ & 0 & 2 \\
\hline Superficial punctate keratopathy & $1(0.4 \%)$ & 0 & 1 \\
\hline Lenses & $13(5.2 \%)$ & 2 & 11 \\
\hline Cataract & $7(2.8 \%)$ & 0 & 7 \\
\hline Presbyopia & $4(1.6 \%)$ & 2 & 2 \\
\hline Posterior synechiae & $1(0.4 \%)$ & 0 & 1 \\
\hline Lens dislocation & $1(0.4 \%)$ & 0 & 1 \\
\hline Conjunctiva & $12(4.8 \%)$ & 2 & 10 \\
\hline Acute conjunctivitis & $6(2.4 \%)$ & 2 & 4 \\
\hline Conjunctival hemorrhage & $6(2.4 \%)$ & 0 & 6 \\
\hline Anterior chamber & $7(2.8 \%)$ & 1 & 6 \\
\hline Glaucoma & $6(2.4 \%)$ & 1 & 5 \\
\hline Hyphema & $1(0.4 \%)$ & 0 & 1 \\
\hline Pupil & $2(0.8 \%)$ & 0 & 2 \\
\hline Pupillary margin degeneration & $1(0.4 \%)$ & 0 & 1 \\
\hline Anisocoria & $1(0.4 \%)$ & 0 & 1 \\
\hline Sclera & $1(0.4 \%)$ & 1 & 0 \\
\hline Episclertitis (infectious) & $1(0.4 \%)$ & 1 & 0 \\
\hline Posterior segment disease & $112(44.4 \%)$ & 45 & 67 \\
\hline Retina & $107(42.5 \%)$ & 45 & 62 \\
\hline CMV retinitis & $35(13.9 \%)$ & 22 & 13 \\
\hline Noninfectious retinopathy & $35(13.9 \%)$ & 14 & 21 \\
\hline HIV retinopathy (Fig. 2) & $23(9.1 \%)$ & 11 & 12 \\
\hline DM retinopathy & $9(3.6 \%)$ & 2 & 7 \\
\hline Retinal hemorrhage & $1(0.4 \%)$ & 0 & 1 \\
\hline HTN retinopathy & $1(0.4 \%)$ & 1 & 0 \\
\hline Sickle cell retinopathy & $1(0.4 \%)$ & 0 & 1 \\
\hline Retinal detachment & $19(7.6 \%)$ & 5 & 14 \\
\hline Retinal detachment & $16(6.3 \%)$ & 4 & 12 \\
\hline Proliferative vitreoretinopathy & $2(0.8 \%)$ & 0 & 2 \\
\hline Retinal tear & $1(0.4 \%)$ & 1 & 0 \\
\hline Necrotizing herpetic retinitis/viral retinitis & $15(5.6 \%)$ & 4 & 11 \\
\hline Cryptococcal retinitis & $1(0.4 \%)$ & 0 & 1 \\
\hline Lattice degeneration & $1(0.4 \%)$ & 0 & 1 \\
\hline Macular hole & $1(0.4 \%)$ & 0 & 1 \\
\hline Vitreous & $1(0.4 \%)$ & 0 & 1 \\
\hline Vitreous syneresis & $1(0.4 \%)$ & 0 & 1 \\
\hline
\end{tabular}


TABle 4. (Continued)

\begin{tabular}{|c|c|c|c|}
\hline Eye anatomic location & All diagnoses $(\mathrm{n}=252)$ & Early ART $(\mathrm{n}=77)$ & Late ART $(\mathrm{n}=175)$ \\
\hline Choroid & $2(0.8 \%)$ & 0 & 2 \\
\hline Choroidal hemorrhage & $1(0.4 \%)$ & 0 & 1 \\
\hline Choroidal detachment & $1(0.4 \%)$ & 0 & 1 \\
\hline Retina vessels & $2(0.8 \%)$ & 0 & 2 \\
\hline Retinal vein occlusion & $2(0.8 \%)$ & 0 & 2 \\
\hline Anterior/posterior segment disease & $18(7.1 \%)$ & 6 & 12 \\
\hline Ocular syphilis & $7(2.8 \%)$ & 2 & 5 \\
\hline Panuveitis & $5(1.9 \%)$ & 2 & 3 \\
\hline Retinitis & $2(0.8 \%)$ & 0 & 2 \\
\hline Noninfectious uveitis & $6(2.4 \%)$ & 3 & 3 \\
\hline Uveitis & $1(0.4 \%)$ & 0 & 1 \\
\hline Behcet's uveitis & $1(0.4 \%)$ & 0 & 1 \\
\hline Iritis & $1(0.4 \%)$ & 1 & 0 \\
\hline Vitritis & $2(0.8 \%)$ & 1 & 1 \\
\hline Choroiditis & $1(0.4 \%)$ & 1 & 0 \\
\hline Endogenous endophthalmitis (bacterial) & $5(1.9 \%)$ & 1 & 4 \\
\hline Neuro-ophthalmic & $19(7.6 \%)$ & 5 & 14 \\
\hline Cranial nerves/extraocular muscles & $7(2.8 \%)$ & 0 & 7 \\
\hline Cranial nerve palsy & $7(2.8 \%)$ & 0 & 7 \\
\hline Noninfectious & $6(2.4 \%)$ & 0 & 6 \\
\hline Infectious & $1(0.4 \%)$ & 0 & 1 \\
\hline Optic nerve & $5(1.9 \%)$ & 3 & 2 \\
\hline Optic neuritis & $5(1.9 \%)$ & 3 & 2 \\
\hline Infectious & $4(1.6 \%)$ & 2 & 2 \\
\hline Noninfectious & $1(0.4 \%)$ & 1 & 0 \\
\hline Brain & $7(2.8 \%)$ & 2 & 5 \\
\hline Migraine with ocular pain & $3(1.2 \%)$ & 0 & 3 \\
\hline Hemianopsia & $2(0.8 \%)$ & 1 & 1 \\
\hline Encephalitis with photophobia (infectious) & $1(0.4 \%)$ & 1 & 0 \\
\hline Gaze preference (non-infectious) & $1(0.4 \%)$ & 0 & 1 \\
\hline
\end{tabular}

FIG. 1. Cytomegalovirus retinitis. This image was originally published in the Retina Image Bank ASRS. Author, Jeremy B. Gross, MD. Uploaded October 10, 2012. Copyright 2013 American Society of Retina Specialists.

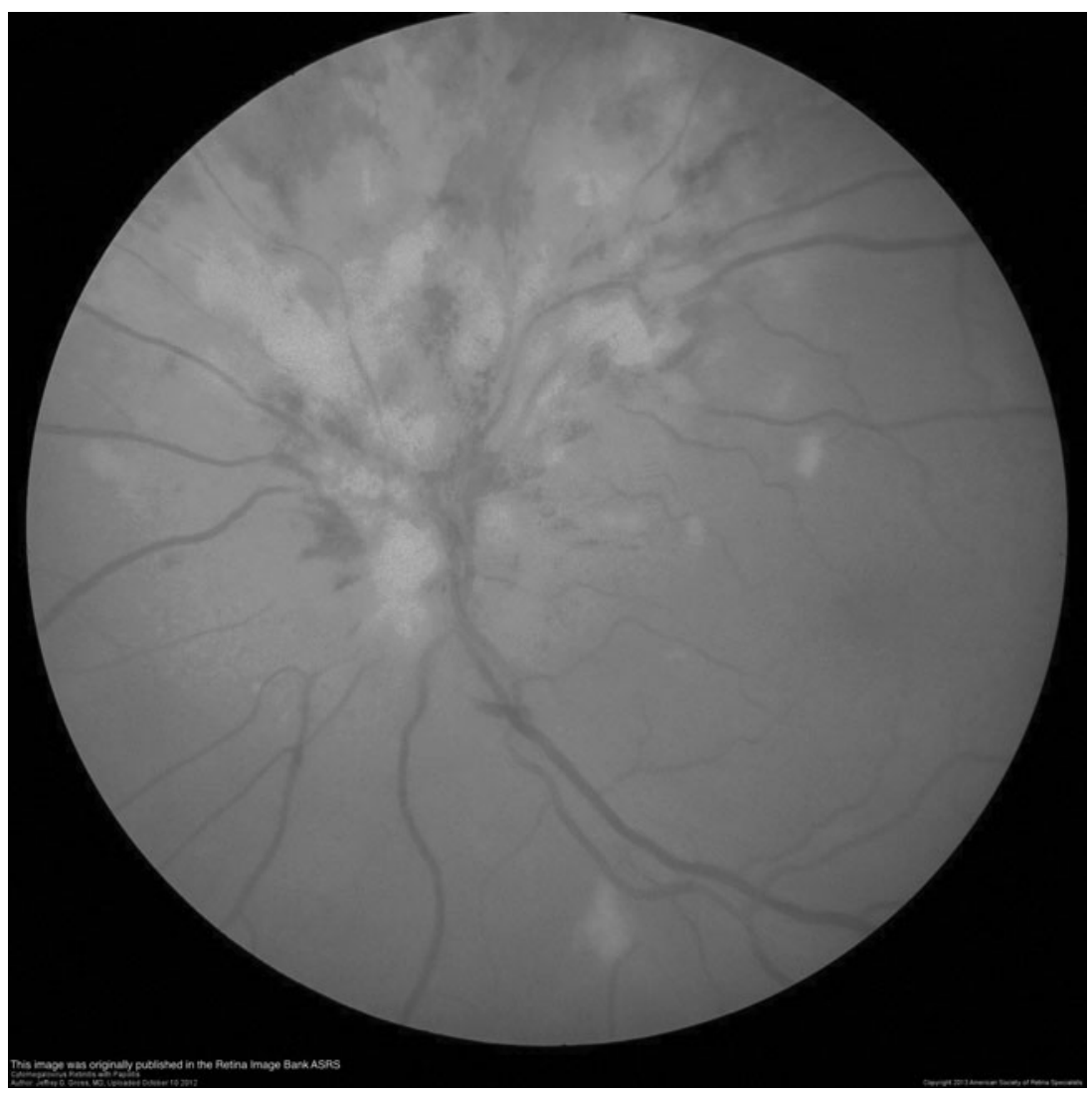




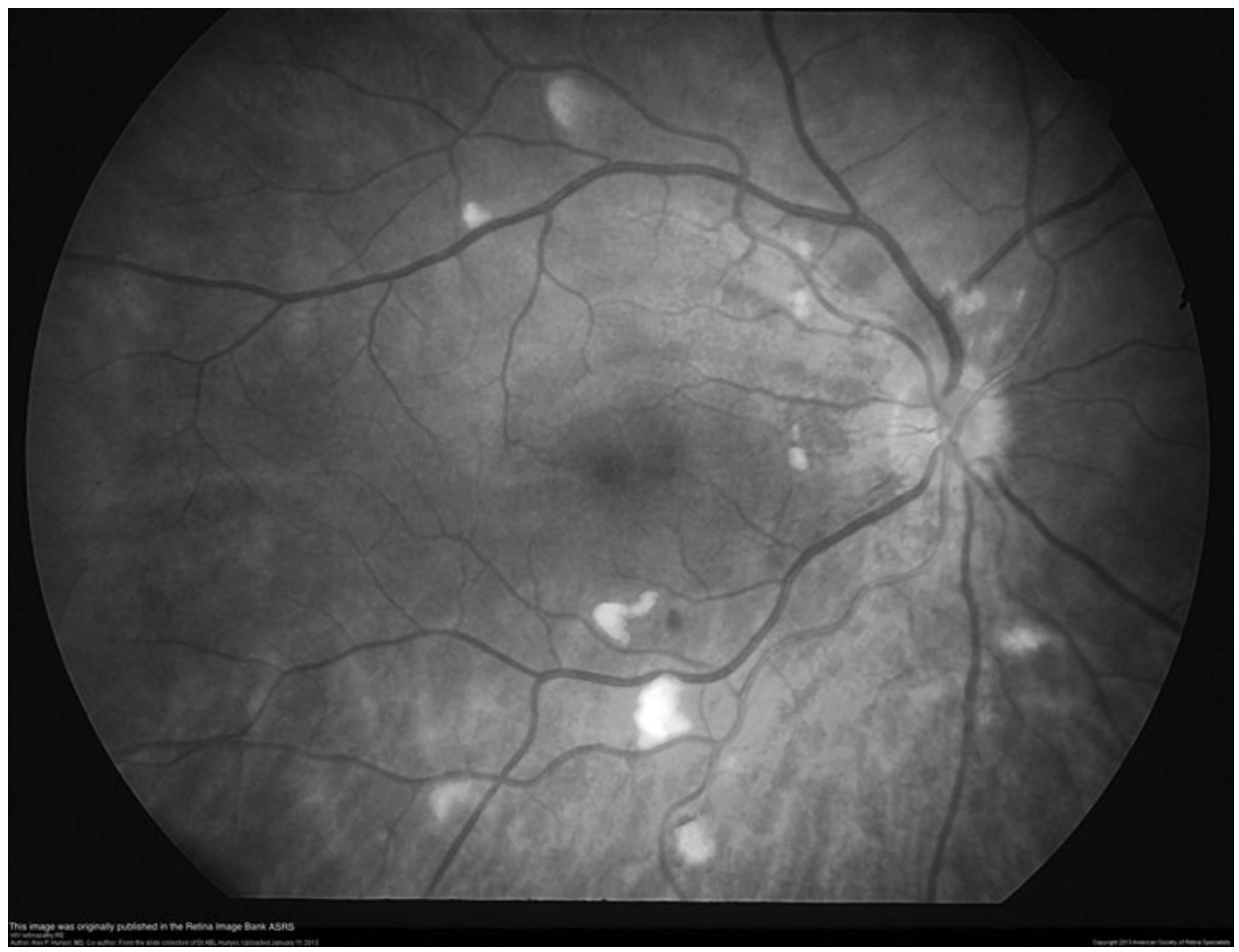

FIG. 2. HIV retinopathy. This image was originally published in the Retina Image Bank ASRS. Author, Alex P. Hunyor, MD. Uploaded January 11, 2013. Copyright 2013 American Society of Retina Specialists.

over this period $^{16}$ (this includes eye diseases such as CMV: several epidemiologic studies of the late 1990's estimate a 50-80\% decrease in the incidence of CMV retinitis alone since the introduction of $\mathrm{ART}^{17-19}$ ). Consequently, the incidence of non-AIDSrelated mortality significantly increased over this time period. ${ }^{2-7}$ Today's HIV practitioners often face their most significant challenges from noninfectious co-morbidities such as coronary artery disease, osteoporosis, chronic kidney disease, hyperlipidemia, liver disease, and non-AIDS defining malignancies.

In our study, noninfectious eye disease was more common overall than infectious disease. In the early ART group, however, infections represented the majority of eye diseases that were seen. The significant decrease in opportunistic in- fections in the late ART group supports the conclusion that ART may have been a less effective intervention in the early ART group. The median time since HIV diagnosis in the early and late groups was 6 and 7.5 years, respectively. Despite an average year and a half added exposure to HIV infection, late ART patients were far less likely to have had an opportunistic infection. One could subsequently conclude that improved efficacy of ART in the late group directly impacted the incidence of infectious eye diseases.

A bias towards an increased number of opportunistic infections in the early ART group may very well exist in this study, however. Many patients in the early ART group may have acquired their opportunistic infections prior to the

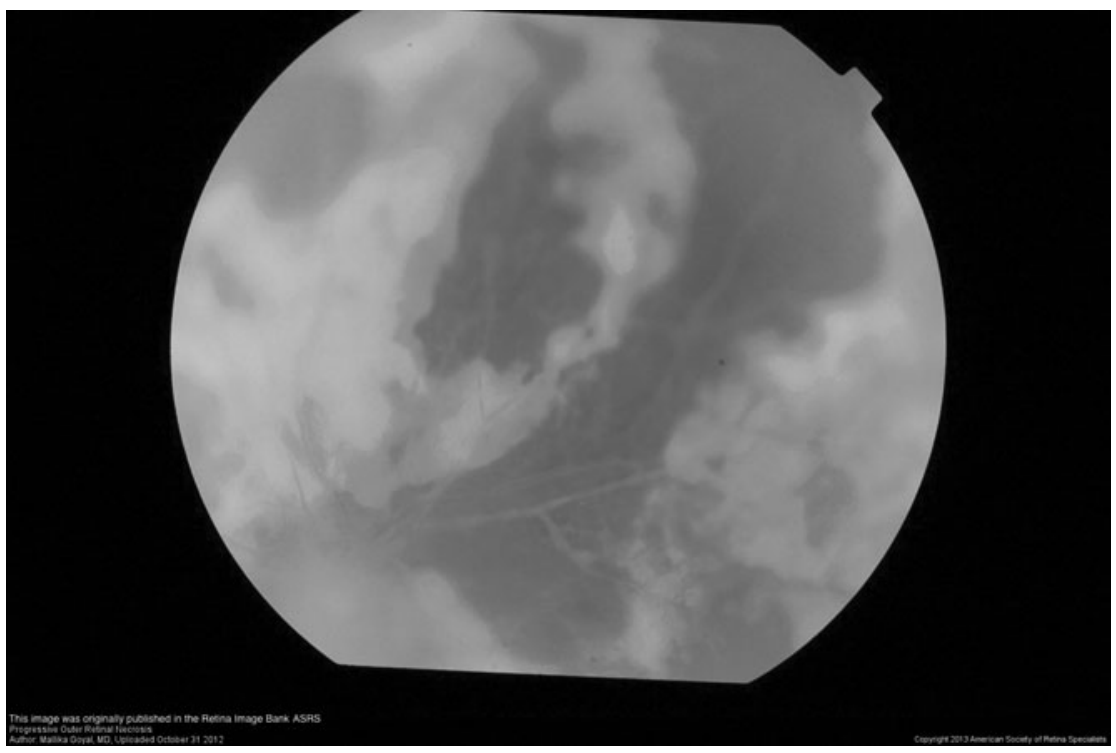

FIG. 3. Progressive outer retinal necrosis. This image was originally published in the Retina Image Bank ASRS. Author, Mallika Goyal, MD. Uploaded October 31, 2012. Copyright 2013 American Society of Retinal Specialists. 


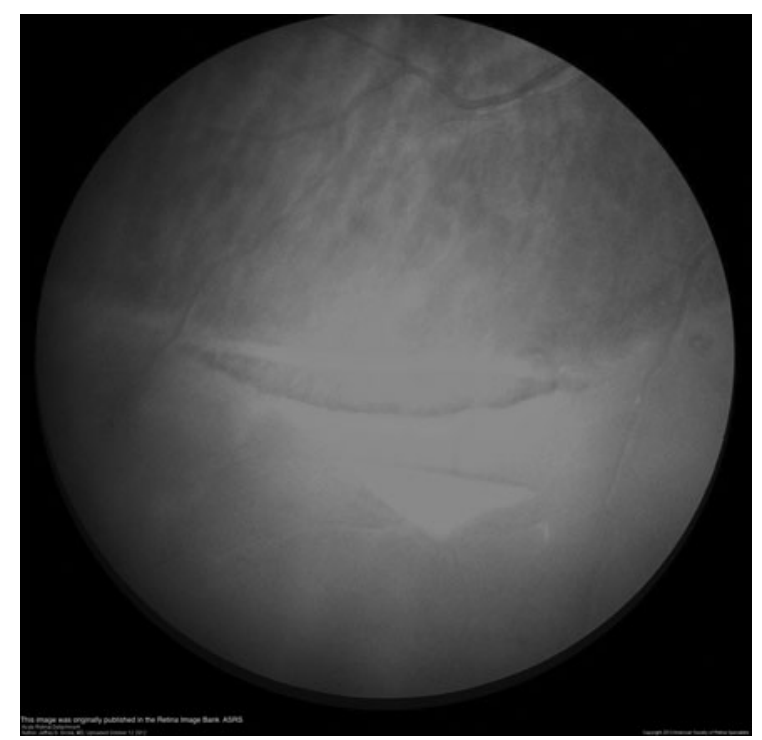

FIG. 4. Acute retinal detachment. This image was originally published in the Retina Image Bank ASRS. Author, Jeffrey G. Gross, MD. Uploaded October 12, 2012. Copyright 2012 American Society of Retina Specialists.

availability of any effective antiretrovirals. They may have subsequently survived long enough to be included in the early ART group. Many such patients may not have survived long enough, however, to be included in the late ART group. A better understanding of HIV treatment and prevention of opportunistic infections may have prevented acquisition of such infections for late ART patients and may partially ac- count for the decreased prevalence of opportunistic infections in the late ART group.

An additional caveat exists in that HIV eye diseases that were frequently managed in the inpatient setting in the 1990s can now be managed in the outpatient setting. For example, a regimen of oral valganciclovir in combination with ART could now potentially be used to treat CMV retinitis in the outpatient setting. However, such a diagnosis would almost universally prompt an inpatient admission in the time frame captured by the early ART group. As this study only examined eye diseases in inpatients, fewer patients with true infectious eye disease may have been captured in the late ART group, since these patients may have been able to avoid hospital admission altogether.

In addition, lack of initial insurance reimbursement for PIs made their availability to patients impractical in many cases until the late 1990s. Therefore, despite the existence of PIs throughout the early ART time period, these effective HIV medications may not have been widely employed over that same time frame, and opportunistic infections may therefore have developed with greater frequency in this group.

The majority of patients with ocular disease, whether infectious or not, experienced diminished visual acuity (when documented). Patients in the late ART group were significantly more likely to have documented visual acuity $(<20 /$ 200) as compared to early ART patients. Our data concur with other larger studies ${ }^{10}$ that also illustrate that visual impairment remains a significant problem in the HIV population, despite improved control of HIV. Emerging noninfectious eye diseases in HIV patients are likely to continue to have an impact on vision, despite improved control of HIV. HIV practitioners, who may be unaware of the potential effects of

FIG. 5. Syphilitic maculopathy. This image was originally published in the Retina Image Bank ASRS. Author, Matthew W. MacCumber, MD, PhD; co-author, Milad Hakimbashi, MD. Photographer, Tara Farmer. Uploaded August 31, 2012. Copyright 2013 American Society of Retina Specialists.

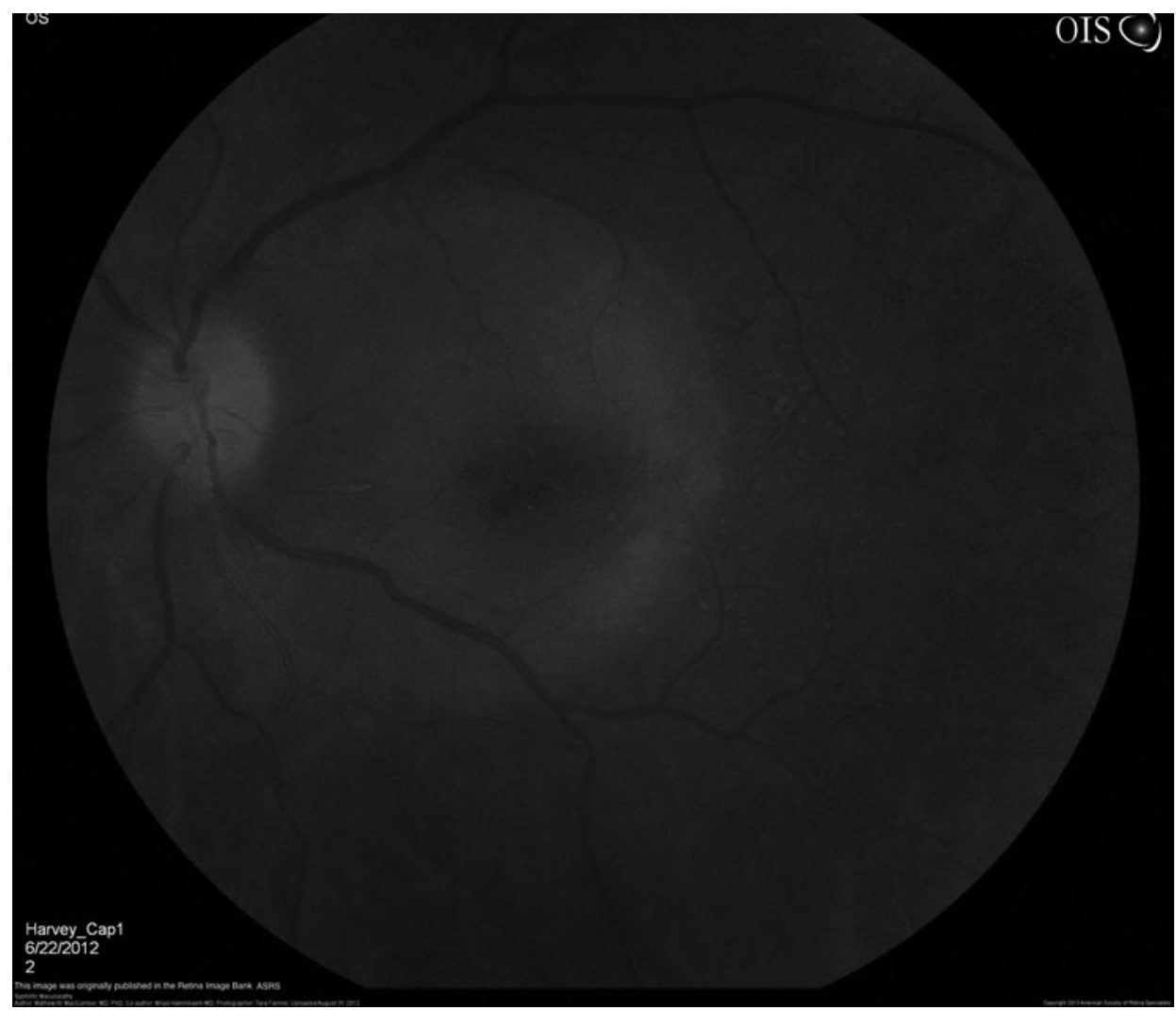




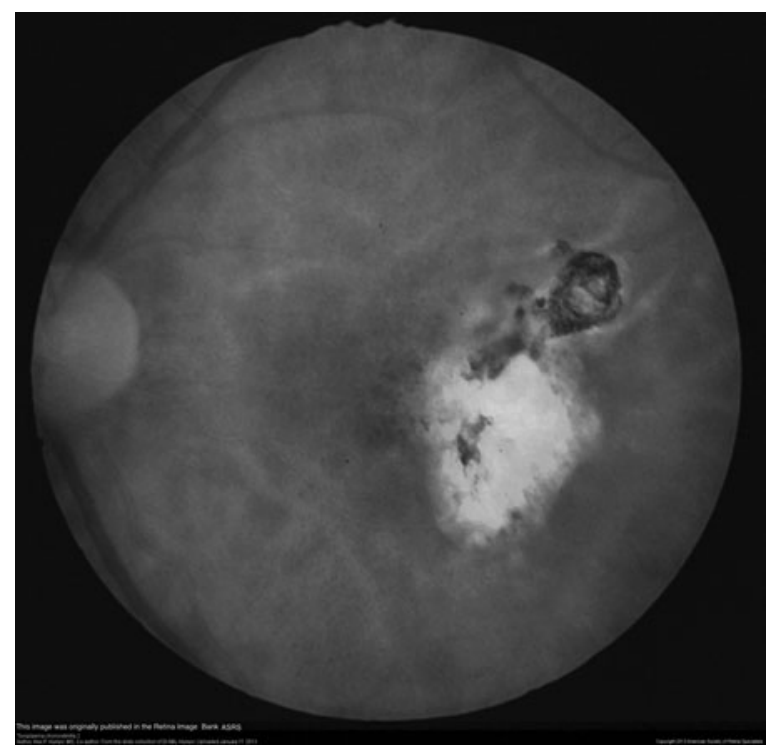

FIG. 6. Toxoplasma chorioretinitis. This image was originally published in the Retina Image Bank ASRS. Author, Alex P. Hunyor, MD. Uploaded January 11, 2013. Copyright 2013 American Society of Retina Specialists.

noninfectious causes of visual impairment, may inadvertently leave evolving eye disease unaddressed.

In conclusion, ART has significantly changed the epidemiologic landscape of HIV disease. The incidence of lifethreatening opportunistic infections has significantly decreased in the setting of newer, more efficacious, and better-tolerated antiretroviral drugs for those individuals who are diagnosed, who are retained in care, and who remain on ART with a suppressed viral load. Noninfectious co-morbidities are often of more pressing concern to the health of HIV patients taking modern ART regimens.

This generalization appears to be true with regard to eye disease in the HIV patient as well. Vision-threatening opportunistic infections such as CMV retinitis were commonly seen prior to the development of antiretroviral medications. Such infections still represented a significant disease burden amongst patients taking early-generation ART regimens; however, it appears that newer iterations of ART are causing a decrease in the incidence of such opportunistic eye infections. Noninfectious eye diseases now appear to be a more significant threat to vision in the late ART HIV patient.

These study data have several limitations. Most notably, this study is a small, cross-sectional examination with an inherent selection bias, since only hospitalized patients were included. These data may not reflect a true representation of eye disease in the general HIV population. Furthermore, patients without subjective eye complaints may have never received an ocular diagnosis, and therefore subclinical eye disease may not have been discovered. Also of note, some demographic information for the patients in this study was unavailable and therefore incomplete.

With these limitations in mind, we conclude that our data provide evidence of a shift in the epidemiology of HIV eye disease. Noninfectious pathology seems to be a more significant cause of visual loss in the late ART era. This observation is consistent with the shifting epidemiology of HIV morbidity and mortality in general and underscores the continued need for routine eye care as a component of the comprehensive management of HIV infected patients.

\section{Acknowledgments}

Data from this study were collected exclusively within the Thomas Jefferson University Hospitals and Wills Eye Institute of Thomas Jefferson University.

\section{Author Disclosure Statement}

There are no commercial conflicts of interest or additional disclosures.

\section{References}

1. Sterling TR, Chaisson RE. General clinical manifestations of human immunodeficiency virus infection (including the acute retroviral syndrome and oral, cutaneous, renal, ocular, metabolic, and cardiac diseases). Mandell, Douglas, and Bennett's Principles and Practice of Infectious Diseases, $7^{\text {th }}$ Ed. Philadelphia, PA: Churchill Livingstone Elsevier, 2010; pp. 1705-1725.

2. Palella FJ, Delaney KM, Moorman AC, et al. Declining morbidity and mortality among patients with advanced immunodeficiency virus infection. N Engl J Med 1998;338:853-860.

3. Mocroft A, Ledergerber B, Katlama C, et al. Decline in the AIDS and death rates in the Eurosida Study: An observational study. Lancet 2003;362:22-27.

4. Mocroft A, Brettle R, Kirk O, et al. Changes in the cause of death among HIV positive subjects across Europe: Results from the Eurosida Study. AIDS 2002;16:1663-1671.

5. Buchacz K, Baker RK, Palella FJ, et al. Rate of opportunistic illnesses and CD4 cell counts at OI diagnosis in a cohort of US patients (1999-2006). Abstract. Conference on Retroviruses and Opportunistic Infections, Montreal, 2009.

6. Egger M, May M, Chene G, et al. Prognosis of HIV-1 infected patients starting highly active antiretroviral therapy: A collaborative analysis of prospective studies. Lancet 2002;360: 119-129.

7. Lemp GF, Payne SF, Neal D, et al. Survival trends for patients with AIDS. JAMA 1990;263:402-406.

8. Jabs DA, Van Natta ML, Holbrook JT, et al. Longitudinal study of the ocular complications of AIDS: Ocular diagnosis at enrollment. Ophthalmology 2007;114:780-786.

9. Freeman WR, Van Natta ML, Jabs D, et al. Vision function in HIV-infected individuals without retinitis; Report of the studies of ocular complications of AIDS Research Group. Am J Ophthalmol 2008;145:453-462.

10. Cunningham Jr ET, Margolis TP. Ocular manifestations of HIV infection. N Engl J Med 1998;339:236-244.

11. Jabs DA. Ocular manifestations of HIV infection. Trans Am Ophthalmol Soc 1995;93:623-683.

12. Sarraf D, Ernest JT. AIDS and the eyes. Lancet 1996;348:525528.

13. Martin DF, Kupperman BD, Wolitz RA, et al. Oral ganciclovir for patients treated with cytomegalovirus retinitis treated with a ganciclovir implant. NEJM 1999;340; 1063-1070.

14. McKenzie R, Travis WD, Dolan SA, et al. The causes of death in patients with human immunodeficiency virus infection: A clinical and pathologic study with emphasis on the role of pulmonary diseases. Medicine (Baltimore) 1991; 70:326-343.

15. Hoover DR, Peng Y, Saah A, et al. Occurrence of cytomegalovirus retinitis after human immunodeficiency vi- 
rus immunosuppression. Arch Ophthalmology 1996;114: 821-827.

16. Palella FJ, Baker RK, Moorman AC, et al. Mortality in the highly active antiretroviral therapy era: Changing causes of death and disease in the HIV Outpatient Study. JAIDS 2006;43:27-34.

17. Jacobson MA, Zegans M, Pavan PR, et al. Cytomegalovirus retinitis after initiation of highly active antiretroviral therapy. Lancet 1997;349:1443-1445.

18. Macdonald JC, Torriani FJ, Morse LS, et al. Lack of reactivation of cytomegalovirus (CMV) retinitis after stopping CMV maintenance therapy in AIDS patients with sustained elevations in CD4 $\mathrm{T}$ cells in response to highly active antiretroviral therapy. J Infect Dis 1998;177:1182-1187.
19. Hammer SM, Squires KE, Hughes MD, et al. A controlled trial of two nucleosides analogues plus indinavir in persons with immunodeficiency virus infection and CD4 cell counts of 200 per cubic millimeter or less. N Engl J Med 1997;337: 725-733.

Address correspondence to: Dr. Christopher Miller Division of Infectious Diseases, Suite 1020 Thomas Jefferson University Hospital 1015 Chestnut Street Philadelphia PA 19107

E-mail: christopher.miller@jeffersonhospital.org 